\title{
Mean curvature flow and Riemannian submersions
}

\author{
Giuseppe Pipoli ${ }^{1}$
}

Received: 2 September 2015 / Accepted: 21 March 2016 / Published online: 26 March 2016

(C) The Author(s) 2016. This article is published with open access at Springerlink.com

\begin{abstract}
We prove that a sufficient condition ensuring that the mean curvature flow commutes with a Riemannian submersion is that the submersion has minimal fibers. We then lift some results taken from the literature (i.e., Andrews and Baker in J Differ Geom 85:357-395, 2010; Baker in The mean curvature flow of submanifolds of high codimension, 2011; Huisken in J Differ Geom 20:237-266, 1984; Math Z 195:205-219, 1987; Pipoli and Sinestrari in Mean curvature flow of pinched submanifolds of $\mathbb{C P}^{n}$ ) to create new examples of evolution by mean curvature flow. In particular we consider the evolution of pinched submanifolds of the sphere, of the complex projective space, of the Heisenberg group and of the tangent sphere bundle equipped with the Sasaki metric.
\end{abstract}

Keywords Mean curvature flow $\cdot$ Riemannian submersions $\cdot$ Pinched submanifolds

Mathematics Subject Classification $53 \mathrm{C} 40 \cdot 53 \mathrm{C} 44$

\section{Introduction}

Let $F_{0}: \mathcal{M} \rightarrow(\overline{\mathcal{M}}, \bar{g})$ be a smooth immersion of an $m$-dimensional manifold into a Riemannian manifold $\overline{\mathcal{M}}$ of dimension $m+k$, called ambient space. We denote by $A$ the second fundamental form and by $H$ the mean curvature vector associated with the immersion. The evolution of $\mathcal{M}_{0}=F_{0}(\mathcal{M})$ by mean curvature flow is the one-parameter family of immersions $F: \mathcal{M} \times\left[0, T_{\max }[\rightarrow \overline{\mathcal{M}}\right.$ satisfying

$$
\left\{\begin{array}{l}
\frac{\partial}{\partial t} F(p, t)=H, \quad p \in \mathcal{M}, \quad t \geq 0, \\
F(\cdot, 0)=F_{0} .
\end{array}\right.
$$

Giuseppe Pipoli

giuseppe.pipoli@ujf-grenoble.fr

1 UMR 5582, CNRS-UJF, Institut Fourier, Université Joseph Fourier (Grenoble I), 100 rue des maths, BP 74, 38402 Saint-Martin-d'Hères, France 
It is well known that if $\mathcal{M}_{0}$ is closed, then this problem has a uniquely defined smooth solution up to some maximal time $T_{\max } \leq \infty$. We frequently identify the immersion $F(., t)$ with the immersed submanifold $\mathcal{M}_{t}=F(\mathcal{M}, t)$.

Let $\left(\mathcal{M}, g_{\mathcal{M}}\right)$ and $\left(\mathcal{B}, g_{\mathcal{B}}\right)$ be two Riemannian manifolds of dimension $m$ and $b$ respectively. A Riemannian submersion is a smooth map $\pi: \mathcal{M} \rightarrow \mathcal{B}$ satisfying the following axioms $S 1$ and $S 2$.

S1) $\pi$ has maximal rank;

For every $p \in \mathcal{M}, \pi^{-1}(p)$ is a submanifold of $\mathcal{M}$ called the fiber over $p$. A vector field on $\mathcal{M}$ is called vertical if it is always tangent to fibers, horizontal if always orthogonal to fibers. The second axiom is

S2) for every $X, Y$ horizontal vectors we have

$$
g_{\mathcal{M}}(X, Y)=g_{\mathcal{B}}\left(\pi_{*} X, \pi_{*} Y\right) \circ \pi .
$$

$\mathcal{M}$ is called the total space of the submersion and $\mathcal{B}$ is called the base. Axiom $S 1$ implies that $m \geq b$ and so the dimension of the fibers is $\hat{m}=m-b$. Axiom $S 2$ says that $\pi$ preserves lengths of horizontal vectors.

The first theorem proved in this paper explores the symmetries of the mean curvature flow and gives a sufficient condition ensuring that this flow commutes with a submersion. We consider submersions defined by the action of a group of isometries. Let $G$ be a Lie group acting as isometries of a Riemannian manifold $\left(\overline{\mathcal{M}}, g_{\overline{\mathcal{M}}}\right)$. Suppose that the quotient space, obtained by identifying the points of an orbit of the action of $G$, is a smooth manifold $\overline{\mathcal{B}}=\overline{\mathcal{M}} / G$, and consider the induced metric $g_{\overline{\mathcal{B}}}$ on it. The natural projection $\pi: \overline{\mathcal{M}} \rightarrow \overline{\mathcal{B}}$ is a Riemannian submersion with fibers the orbits of $G$. If the action of $G$ is free we have the wellknown principal bundles. In this case the fibers of $\pi$ are isometric to the group $G$. The best known examples of such submersions are probably the Hopf fibrations: $\pi_{1}: \mathbb{S}^{2 n+1} \rightarrow \mathbb{C P}^{n}$ and $\pi_{2}: \mathbb{S}^{4 n+3} \rightarrow \mathbb{H} \mathbb{P}^{n}$.

Lifting a submanifold of $\overline{\mathcal{B}}$ we have a $G$-invariant submanifold of $\overline{\mathcal{M}}$; vice versa, projecting a $G$-invariant submanifold of $\overline{\mathcal{M}}$ we get a submanifold of $\overline{\mathcal{B}}$. We want to study how the mean curvature flow is related to a submersion.

Theorem 1.1 Let $\pi: \overline{\mathcal{M}} \rightarrow \overline{\mathcal{B}}=\overline{\mathcal{M}} / G$ be a Riemannian submersion. If $\pi$ has closed and minimal fibers then the mean curvature flow of any closed submanifold commutes with the submersion. More precisely, let $\mathcal{M}_{0}$ be a $G$-invariant submanifold of $\overline{\mathcal{M}}$ and $\mathcal{B}_{0}=\pi\left(\mathcal{M}_{0}\right)$; therefore the mean curvature flow of $\mathcal{M}_{0}$ and $\mathcal{B}_{0}$ are defined up to the same maximal time $T_{\max }$ and $\pi\left(\mathcal{M}_{t}\right)=\mathcal{B}_{t}$ for any time $0 \leq t<T_{\max }$.

Note that closedness of fibers and of the initial immersions guarantees the uniqueness of the solution of mean curvature flow of the submanifold $\mathcal{B}_{0}$ and its lift. The mean curvature flow in manifolds with symmetries has been studied by several authors, for example Pacini in [14] considered the evolution of the orbits of a group of isometries. The proof of Theorem 1.1 is based on the fundamental equations for submersions which are derived in the classical paper by O'Neill [13]. Although similar computations already appear in the previous literature on geometric flows, see in particular the paper [16], it seems to us that this result has never been explicitly observed before. The main part of this paper is devoted to the applications to specific examples, where we obtain new convergence results for the mean curvature flow by lifting the known theorems for the base manifold to the ambient space.

For example consider the main theorem of [15]: it concerns evolution by mean curvature flow of pinched submanifolds of $\mathbb{C P}^{n}$. Lifting this result with Theorem 1.1 applied to the 
Hopf fibration $\pi: \mathbb{S}^{2 n+1} \rightarrow \mathbb{C P}^{n}$, we have the following new examples of evolution of pinched hypersurfaces of the sphere.

Proposition 1.2 Let $\mathcal{M}_{0}$ be a closed $\mathbb{S}^{1}$-invariant hypersurface of $\mathbb{S}^{2 n+1}(c)$, the sphere of constant sectional curvature $c>0$ with $n \geq 3$. If $\mathcal{M}_{0}$ satisfies

$$
|A|^{2}<\frac{1}{2 n-2}|H|^{2}+4 c,
$$

then the mean curvature flow of $\mathcal{M}_{0}$ develops a singularity in finite time and converges to a $\mathbb{S}^{1}$, therefore such an $\mathcal{M}_{0}$ is diffeomorphic to a $\mathbb{S}^{1} \times \mathbb{S}^{2 n-1}$.

It is interesting to compare this statement with the classical result by Huisken in [9]. The pinching inequality (1.2) is weaker than the one required in [9], but we have the further assumption about the $\mathbb{S}^{1}$-invariance. In fact, we find neither of the two possibilities described by Huisken, i.e. the convergence to a round point in finite time or the convergence to a totally geodesic submanifold in infinite time. Let us also recall the paper [12] by Nguyen, who considered the same pinching condition (1.2) together with positive mean curvature, and proved that the flow develops a cylindrical singularity. Our results is similar, but our assumption of $\mathbb{S}^{1}$-invariance allows to give a more complete description by describing the global behavior of the evolution and not only around a singularity.

Another result of [15] regards the evolution of pinched hypersurfaces of $\mathbb{H}^{n}$. Applying Theorem 1.1 to the Hopf fibration $\pi: \mathbb{S}^{4 n+3} \rightarrow \mathbb{H}^{n}$ we get

Proposition 1.3 Let $\mathcal{M}_{0}$ be a closed $\mathbb{S}^{3}$-invariant hypersurface of $\mathbb{S}^{4 n+3}(c)$, with $n \geq 3$. If $\mathcal{M}_{0}$ satisfies

$$
|A|^{2}<\frac{1}{4 n-2}|H|^{2}+8 c,
$$

then the mean curvature flow of $\mathcal{M}_{0}$ develops a singularity in finite time and converges to an $\mathbb{S}^{3}$, then such an $\mathcal{M}_{0}$ is diffeomorphic to an $\mathbb{S}^{3} \times \mathbb{S}^{4 n-1}$.

Note that $\mathbb{S}^{1}$ is a subgroup of $\mathbb{S}^{3}$, then if a submanifold of $\mathbb{S}^{4 n+3}$ is $\mathbb{S}^{3}$-invariant, we can project it both to $\mathbb{C P}^{2 n+1}$ and $\mathbb{H} \mathbb{P}^{n}$. Putting together Propositions 1.2 and 1.3 we obtain a negative result.

Corollary 1.4 There are no closed $\mathbb{S}^{3}$-invariant hypersurfaces of $\mathbb{S}^{4 n+3}(c)$ such that

$$
|A|^{2}<\frac{1}{4 n}|H|^{2}+4 c .
$$

Propositions 1.2 and 1.3 just discussed are proved in a more general setting: we deform the metric of the sphere using the canonical variations of the Hopf fibrations. After that some other examples are described. Lifting the main result of [15] in the case of higher codimension, we get Proposition 4.2. It concerns $\mathbb{S}^{1}$-invariant pinched submanifolds of higher codimension of the sphere. We find the alternative between the convergence in finite time to an $\mathbb{S}^{1}$ and the convergence in infinite time to a totally geodesic submanifold, which is an $\mathbb{S}^{m}$ for some $m$. In particular the second case can occur only if the dimension of the evolving submanifold is even. This result generalizes the one of Baker [2]. Starting again from [15] we can prove Proposition 4.4. It is about pinched hypersurfaces of the complex projective space and extends [15] itself. The ambient space is the Heisenberg group in Propositions 4.5 and 4.6, which are derived from the results of mean curvature flow in the Euclidean space, i.e. [1,8]. Finally in Proposition 4.8 we study submanifolds of the tangent sphere bundle of the round sphere 
equipped with the Sasaki metric and prove the alternative between the convergence in finite time to an orbit and the convergence in infinite time to a minimal, but not totally geodesic, limit. This result is obtained by lifting the classical result of Huisken [9] regarding pinched hypersurfaces of the sphere and its generalization [2] to arbitrary codimension.

The paper is organized as follows. In Sect. 2 we recall some notation and preliminary results, in particular we compute the relationships between the second fundamental forms of a submanifold and its lift through a Riemannian submersion. In Sect. 3 first we show that the invariance of a submanifold with respect to a group of isometries of the ambient manifold is preserved by the mean curvature flow, then Theorem 1.1 is proved. In Sect. 4 we have the applications of Theorem 1.1, i.e. Propositions 1.2, 1.3, 4.2, 4.4, 4.5, 4.6 and 4.8 are proved.

\section{Preliminaries}

In this section we recall some basic notions and fix some notations used throughout this paper. Consider $F: \mathcal{M} \rightarrow(\overline{\mathcal{M}}, \bar{g})$ a smooth immersion of an $m$-dimensional differential manifold $\mathcal{M}$ into a Riemannian manifold $\overline{\mathcal{M}}$ of dimension $m+k$. Unless stated otherwise, geometric quantities of the submanifolds are indicated in the usual way, while for the ambient manifold we use a line over the common symbol. Moreover, Latin letters $i, j, k, \ldots$ are related to $T_{x} \mathcal{M}$, the tangent space to $\mathcal{M}$ at $x$, and Greek letters $\alpha, \beta, \gamma, \ldots$ to the normal space $N_{x} \mathcal{M}$. Fix $\left(x_{1}, \ldots, x_{n}\right)$ a local coordinate system around a point $x \in \mathcal{M}$. The local expression of $g$ is

$$
g_{i j}(x)=\bar{g}_{F(x)}\left(\frac{\partial F}{\partial x_{i}}, \frac{\partial F}{\partial x_{j}}\right)
$$

Let $\bar{\nabla}$ be the Levi-Civita connection of $(\overline{\mathcal{M}}, \bar{g})$. The second fundamental form $A$ of the immersion $F$ is defined for every $X, Y$ tangent vectors of $\mathcal{M}$ by

$$
A(X, Y)=\left(\bar{\nabla}_{X} Y\right)^{\perp}
$$

where $\perp$ denotes the component normal to $\mathcal{M}$.

Let $\left(\xi_{1}, \ldots, \xi_{k}\right)$ be an orthonormal frame of $N_{x} \mathcal{M}$ : the second fundamental form can be written

$$
A=h^{\alpha} \otimes \xi_{\alpha},
$$

where the $h^{\alpha}=\left(h_{i j}^{\alpha}\right)$ are symmetric $(0,2)$-tensors. Here and in the following, if there are no explicit signs of sum, we use Einstein notation, that is we sum over repeated indices. The metric induces a natural isomorphism between tangent and cotangent space. In coordinates, this is expressed in terms of raising/lowering indices by means of the matrices $g_{i j}$ and $g^{i j}$, where $g^{i j}$ is the inverse of $g_{i j}$. The scalar product on the tangent space extends to any tensor bundle, by contracting any pair of lower and upper indices with $g_{i j}$ and $g^{i j}$ respectively. This also allows us to define the norm of any tensor $T$. A function that we use very often is the norm of the second fundamental form

$$
|A|^{2}=\sum_{\alpha}\left|h^{\alpha}\right|^{2}
$$

The trace with respect to the metric $g$ of the second fundamental form is the mean curvature vector $H$ :

$$
H=\operatorname{tr} A=\operatorname{trh}^{\alpha} e_{\alpha}=g^{i j} h_{i j}^{\alpha} e_{\alpha}
$$


It is independent of the orientation and it is well defined globally even if $\mathcal{M}$ is non-orientable. Note that some authors define the mean curvature as the trace of $A$ over $m$; of course this makes no substantial difference in the analysis.

What follows is taken from the classical O'Neill's paper [13]; many other interesting results about Riemannian submersions can be found in chapter 9 of [3] and in the extensive monograph [6]. Let $\pi:\left(\mathcal{M}, g_{\mathcal{M}}\right) \rightarrow\left(\mathcal{B}, g_{\mathcal{B}}\right)$ be a Riemannian submersion. If not specified otherwise, we use the same symbols for geometric quantities of $\mathcal{M}$ and $\mathcal{B}$. It will be clear from the context which manifold we are in. The same quantities of the fibers are distinguished by the superscript ${ }^{\wedge}$. The vertical distribution $\mathscr{V}$ is the distribution of vertical vector fields, that is, $\mathscr{V}=\operatorname{ker} \pi_{*}$. Its orthogonal complement with respect to $g_{\mathcal{M}}$ is the horizontal distribution $\mathscr{H}$. We denote with the same symbols $\mathscr{H}$ and $\mathscr{V}$ the projections of the tangent space of $\mathcal{M}$ to the subspaces of horizontal and vertical vectors, respectively. Then every $X$ tangent to $\mathcal{M}$ can be decomposed in a unique way into the sum of a horizontal and a vertical vector:

$$
X=\mathscr{H} X+\mathscr{V} X .
$$

A horizontal vector field $X^{\prime}$ is called basic if there exists a vector field $X$ on $\mathcal{B}$ such that $\pi_{*} X^{\prime}=$ $X$ : in this case $X$ and $X^{\prime}$ are said to be $\pi$-related. There is a one-to-one correspondence between basic vector fields on $\mathcal{M}$ and arbitrary vector fields on $\mathcal{B}$ : every basic vector field gives a vector field on $\mathcal{B}$ by definition, while every $X$ tangent to $\mathcal{B}$ has a unique horizontal lift $X^{\mathscr{H}}$ to $\mathcal{M}$ characterized by $\pi_{*} X^{\mathscr{H}}=X$. Submersions are ruled by two tensors. For every $X$ and $Y$ tangent to $\mathcal{M}$ we define

$$
\begin{aligned}
\mathcal{T}_{X} Y & =\mathscr{H} \nabla_{\mathscr{V} X}(\mathscr{V} Y)+\mathscr{V} \nabla_{\mathscr{V} X}(\mathscr{H} Y) \\
\mathcal{A}_{X} Y & =\mathscr{V} \nabla_{\mathscr{H} X}(\mathscr{H} Y)+\mathscr{H} \nabla_{\mathscr{H} X}(\mathscr{V} Y)
\end{aligned}
$$

Note that if $X$ and $Y$ are tangent to fibers, i.e. vertical, then $\mathcal{T}_{X} Y=\hat{A}(X, Y)$, where $\hat{A}$ is the second fundamental form of the fibers as submanifolds of $\mathcal{M}$. We have that $\mathcal{T} \equiv 0$ if and only if each fiber is totally geodesic, while $\mathcal{A} \equiv 0$ if and only if $\mathscr{H}$ is integrable.

Since we deal with the mean curvature flow we want to understand how a submanifold of $\overline{\mathcal{B}}$ is related to its lift to $\overline{\mathcal{M}}$ : let $\pi:(\overline{\mathcal{M}}, g \overline{\mathcal{M}}) \rightarrow\left(\overline{\mathcal{B}}, g_{\overline{\mathcal{B}}}\right)$ be a Riemannian submersion, and $F: \mathcal{B} \rightarrow \overline{\mathcal{B}}$ an immersion. $\pi^{-1}(F(\mathcal{B}))$ is a submanifold of $\overline{\mathcal{M}}$ of the same codimension of $F(\overline{\mathcal{B}})$. Formally there is a manifold $\mathcal{M}$, an immersion $F^{\prime}: \mathcal{M} \rightarrow \overline{\mathcal{M}}$ and a submersion that we indicate again with $\pi$, such that the following diagrams commute:

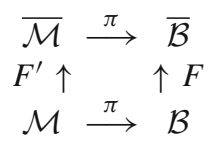

We want to understand the link between $A$, the second fundamental form of $F$, and $A^{\prime}$, the second fundamental form of $F^{\prime}$. Our main tool are the following formulas by O'Neill.

Lemma 2.1 [13] For any tangent vector fields on $\overline{\mathcal{B}} X$ and $Y$ we have

1) $[X, Y]^{\mathscr{H}}=\mathscr{H}\left[X^{\mathscr{H}}, Y^{\mathscr{H}}\right]$;

2) $\left(\bar{\nabla}_{X} Y\right)^{\mathscr{H}}=\mathscr{H}\left(\bar{\nabla}_{X} \mathscr{H} Y^{\mathscr{H}}\right)$.

Lemma 2.2 [13] Let $X$ and $Y$ be horizontal vector fields and $V$ and $W$ vertical vector fields. Then

1) $\bar{\nabla}_{V} W=\mathcal{T}_{V} W+\hat{\nabla}_{V} W$;

2) $\bar{\nabla}_{V} X=\mathscr{H} \bar{\nabla}_{V} X+\mathcal{T}_{V} X$; 
3) $\bar{\nabla}_{X} V=\mathcal{A}_{X} V+\mathscr{V} \bar{\nabla}_{X} V$;

4) $\bar{\nabla}_{X} Y=\mathscr{H} \bar{\nabla}_{X} Y+\mathcal{A}_{X} Y$.

Note that, by construction, $\mathcal{M} \equiv F^{\prime}(\mathcal{M})$ is tangent to the fibers, then any vector normal to $\mathcal{M}$ is necessarily horizontal. From Lemma 2.1 and Gauss equation we have that for any $X$ and $Y$ tangent to $\mathcal{M}$

$$
\begin{aligned}
\bar{\nabla}_{X} \mathscr{H} Y^{\mathscr{H}} & =\mathscr{H}\left(\bar{\nabla}_{X} \mathscr{H} Y^{\mathscr{H}}\right)+\mathscr{V}\left(\bar{\nabla}_{X} \mathscr{H} Y^{\mathscr{H}}\right) \\
& =\left(\bar{\nabla}_{X} Y\right)^{\mathscr{H}}+\mathscr{V}\left(\bar{\nabla}_{X} \mathscr{H} Y^{\mathscr{H}}\right) \\
& =\left(\nabla_{X} Y\right)^{\mathscr{H}}+(A(X, Y))^{\mathscr{H}}+\mathscr{V}\left(\bar{\nabla}_{X} \mathscr{H} Y^{\mathscr{H}}\right) .
\end{aligned}
$$

By definition $A^{\prime}\left(X^{\mathscr{H}}, Y^{\mathscr{H}}\right)=\left(\bar{\nabla}_{X} \mathscr{H} Y^{\mathscr{H}}\right)^{\perp}$ (the component normal to $\mathcal{M}$ ), thus it is a horizontal vector field. By (2.1) we have

$$
A^{\prime}\left(X^{\mathscr{H}}, Y^{\mathscr{H}}\right)=\left(\left(\nabla_{X} Y\right)^{\mathscr{H}}\right)^{\perp}+\left((A(X, Y))^{\mathscr{H}}\right)^{\perp} .
$$

The vector field $\left(\nabla_{X} Y\right)^{\mathscr{H}}$ is the lift of a vector field tangent to $\mathcal{B}$, and so it is tangent to $\mathcal{M}$. In the same way $(A(X, Y))^{\mathscr{H}}$ is normal to $\mathcal{M}$. Hence we have

$$
A^{\prime}\left(X^{\mathscr{H}}, Y^{\mathscr{H}}\right)=(A(X, Y))^{\mathscr{H}} .
$$

Now consider two vertical vector fields $V$ and $W$. They are tangent to $\mathcal{M}$ by construction. $A^{\prime}(V, W)$ is normal to $\mathcal{M}$ and so it is a horizontal vector field. By Lemma 2.2 we have

$$
A^{\prime}(V, W)=\left(\bar{\nabla}_{V} W\right)^{\perp}=\left(\mathscr{H} \bar{\nabla}_{V} W\right)^{\perp}=\left(\mathcal{T}_{V} W\right)^{\perp}=(\hat{A}(V, W))^{\perp} .
$$

Lemma 2.2 does not say anything about the mixed terms $A^{\prime}(X, V)$ with $X$ horizontal and $V$ vertical: they strongly depend on the specific submersion considered as we will see in the examples of Sect. 4.

Notation 2.3 For any submersion $\pi$ considered below $\left(X_{1}, \ldots, X_{m}\right)$ denotes a local orthonormal frame tangent to a submanifold of the base space around a point $p$ and $\left(V_{1}, \ldots, V_{\hat{m}}\right)$ is a local orthonormal set of vertical vector fields. Then around any point $q$ of the fiber $\pi^{-1}(p)$ we use the orthonormal basis $\left(X_{1}^{\mathscr{H}}, \ldots, X_{m}^{\mathscr{H}}, V_{1}, \ldots V_{\hat{m}}\right)$ tangent to the lift of the submanifold. Moreover $\left(\xi_{1}, \ldots, \xi_{k}\right)$ is a local orthonormal frame normal to a submanifold of the base, hence $\left(\xi_{1}^{\mathscr{H}}, \ldots, \xi_{k}^{\mathscr{H}}\right)$ is a local orthonormal frame normal to the lift of the submanifold considered.

Summarizing what we have found, we have

$$
A^{\prime}=\left(\begin{array}{c|c}
h_{i j}^{\mathscr{H}} & \text { mixed terms } \\
\hline \text { mixed terms } & \hat{h}_{i j}^{\perp}
\end{array}\right)
$$

where $h_{i j}=A\left(X_{i}, X_{j}\right)$ and $\hat{h}_{i j}=\hat{A}\left(V_{i}, V_{j}\right)$.

Starting from a fixed Riemannian submersion $\pi:\left(\mathcal{M}, g_{\mathcal{M}}\right) \rightarrow\left(\mathcal{B}, g_{\mathcal{B}}\right)$ there is a standard way to deform the metric $g_{\mathcal{M}}$ to obtain another Riemannian submersion. The canonical variation of $g_{\mathcal{M}}$ is the family of metrics $\left\{g_{\lambda}\right\}_{\lambda>0}$ on $\mathcal{M}$ such that

$$
\begin{array}{ll}
g_{\lambda}(U, V)=\lambda g_{\mathcal{M}}(U, V) & \text { if } U, V \in \mathscr{V}, \\
g_{\lambda}(X, Y)=g_{\mathcal{M}}(U, V) & \text { if } X, Y \in \mathscr{H}, \\
g_{\lambda}(U, X)=0 & \text { if } U \in \mathscr{V}, X \in \mathscr{H} .
\end{array}
$$


Obviously $g_{1}=g_{\mathcal{M}}$. For any $\lambda>0, g_{\lambda}$ makes $\pi$ a Riemannian submersion with the same horizontal and vertical distributions and the same fibers. Let $\nabla^{\lambda}$ be the Levi-Civita connection of the metric $g_{\lambda}$. A straightforward computation gives:

$$
\begin{aligned}
\mathscr{V}\left(\nabla_{U}^{\lambda} V\right) & =\mathscr{V}\left(\nabla_{U}^{1} V\right), \quad \mathscr{H}\left(\nabla_{U}^{\lambda} V\right)=\lambda \mathscr{H}\left(\nabla_{U}^{1} V\right), \\
\nabla_{X}^{\lambda} U & =\nabla_{X}^{1} U, \quad \nabla_{U}^{\lambda} X=\nabla_{U}^{1} X, \quad \nabla_{X}^{\lambda} Y=\nabla_{X}^{1} Y,
\end{aligned}
$$

for every $U, V \in \mathscr{V}$ and $X, Y \in \mathscr{H}$. It follows that $\pi:\left(\mathcal{M}, g_{\mathcal{M}}=g_{1}\right) \rightarrow\left(\mathcal{B}, g_{\mathcal{B}}\right)$ has minimal (resp. totally geodesic) fibers if and only if $\pi:\left(\mathcal{M}, g_{\lambda}\right) \rightarrow\left(\mathcal{B}, g_{\mathcal{B}}\right)$ has minimal (resp. totally geodesic) fibers for every $\lambda>0$. Moreover let $\left(V_{1}, \ldots V_{\hat{m}}\right)$ be a local $g_{\mathcal{M}}$-orthonormal set of vertical vectors: then for any $\lambda>0\left(\lambda^{-\frac{1}{2}} V_{1}, \ldots \lambda^{-\frac{1}{2}} V_{\hat{m}}\right)$ is $g_{\lambda}$-orthonormal. Using (2.5), it is easy to see that, with respect to this basis, the Eq. (2.4) becomes

$$
A_{\lambda}^{\prime}=\left(\begin{array}{c|c}
h_{i j}^{\mathscr{H}} & \lambda^{-\frac{1}{2}} \text { mixed terms } \\
\hline \lambda^{-\frac{1}{2}} \text { mixed terms } & \hat{h}_{i j}^{\perp}
\end{array}\right)
$$

\section{Symmetries of the mean curvature flow}

In this section we prove Theorem 1.1.

Lemma 3.1 Let $F_{0}: \mathcal{M} \rightarrow \overline{\mathcal{M}}$ be a closed immersion and $\varphi$ an isometry of $\overline{\mathcal{M}}$ : then $\varphi$ commutes with the mean curvature flow. Formally if $G_{0}=\varphi \circ F_{0}$ and $F_{t}$ and $G_{t}$ are the evolutions of $F_{0}$ and $G_{0}$ respectively, we have that $G_{t}=\varphi \circ F_{t}$ for any time $t$ for which the flow is defined.

Proof Since $\varphi$ is an isometry we have

$$
\frac{\partial}{\partial t}\left(\varphi \circ F_{t}\right)(p, t)=\varphi_{*} H^{F}(p, t)=H^{\varphi \circ F}(p, t),
$$

where $H^{\psi}$ is the mean curvature vector of $\psi$ for any immersion $\psi$. Then $\varphi \circ F_{t}$ is a solution of the mean curvature flow of initial data $\varphi \circ F_{0}=G_{0}$. Due to the uniqueness of the solution we have the thesis.

It follows immediately that

Corollary 3.2 Let $F_{0}$ and $\varphi$ be defined as in Lemma 3.1 and let $G$ be a group of isometries of $\overline{\mathcal{M}}$. We have

1) if $F_{0}$ is $\varphi$-invariant, then $F_{t}$ is $\varphi$-invariant for any $t$,

2) if $F_{0}$ is $G$-invariant then $F_{t}$ is $G$-invariant for every time $t$.

Proof of Theorem 1.1. Let $F_{0}: \mathcal{B} \rightarrow \overline{\mathcal{B}}$ and $F_{0}^{\prime}: \mathcal{M} \rightarrow \overline{\mathcal{M}}$ two immersions for $\mathcal{B}_{0}$ and $\mathcal{M}_{0}$ respectively. By hypothesis we have that $F_{0}^{\prime}$ is $G$-invariant and $\pi \circ F_{0}^{\prime}=F_{0} \circ \pi$. The crucial point is that, since the fibers are minimal, we have that $H^{\prime}$ is basic and is $\pi$-related to $H$, where $H$ is the mean curvature vector of any submanifold of $\overline{\mathcal{B}}$, and $H^{\prime}$ is the mean curvature vector of its lift to $\overline{\mathcal{M}}$. In fact $H^{\prime}$ is horizontal because it is normal to $\mathcal{M}$. Using the notation of (2.4) we have 


$$
\begin{aligned}
H^{\prime}=\operatorname{tr} A^{\prime} & =\sum_{i} A^{\prime}\left(X_{i}^{\mathscr{H}}, X_{i}^{\mathscr{H}}\right)+\sum_{i} A^{\prime}\left(V_{i}, V_{i}\right) \\
& =\sum_{i} A\left(X_{i}, X_{i}\right)^{\mathscr{H}}+\sum_{i} \hat{A}\left(V_{i}, V_{i}\right)^{\perp} \\
& =\left(\sum_{i} A\left(X_{i}, X_{i}\right)\right)^{\mathscr{H}}+\left(\sum_{i} \hat{A}\left(V_{i}, V_{i}\right)\right)^{\perp} \\
& =H^{\mathscr{H}}+\hat{H}^{\perp} .
\end{aligned}
$$

If the fibers are minimal we get $H^{\prime}=H^{\mathscr{H}}$, that is, $H^{\prime}$ and $H$ are $\pi$-related. In particular $\pi_{*} H^{\prime}=H$ holds. Now let $F_{t}$ be the evolution of $F_{0}, F_{t}^{\prime}$ the lift of $F_{t}, \widetilde{F}_{t}^{\prime}$ the evolution of $F_{0}^{\prime}$ and $\widetilde{F}_{t}$ the projection of $\widetilde{F}_{t}^{\prime}$, and let $H, H^{\prime}, \widetilde{H}^{\prime}$ and $H$ be the respective mean curvature vectors. By construction we have that for any $t$

$$
\pi \circ F_{t}^{\prime}=F_{t} \circ \pi
$$

and $F_{t}^{\prime}$ is $G$-invariant. Then in particular $H^{\prime}$ is horizontal. Differentiating (3.1) we have

$$
\pi_{*} \frac{\partial}{\partial t} F_{t}^{\prime}=\frac{\partial}{\partial t}\left(F_{t} \circ \pi\right)=H=\pi_{*} H^{\prime}
$$

Then $\frac{\partial}{\partial t} F_{t}^{\prime}=H^{\prime}+V^{\prime}$ for some vertical vector field $V^{\prime}$. Since $F_{t}^{\prime}$ is $G$-invariant, $V^{\prime}$ is tangent to $F_{t}^{\prime}\left(\mathcal{M}^{\prime}\right)$. Therefore

$$
\left(\frac{\partial}{\partial t} F_{t}^{\prime}\right)^{\perp}=H^{\prime} .
$$

This means that, up to a tangential diffeomorphism, $F_{t}^{\prime}$ is the solution of the mean curvature flow of initial data $F_{0}^{\prime}$. Then $F_{t}^{\prime}(\mathcal{M})=\widetilde{F}_{t}^{\prime}(\mathcal{M})$ for every time $t$. Vice versa,

$$
\frac{\partial}{\partial t}\left(\widetilde{F}_{t} \circ \pi\right)=\frac{\partial}{\partial t}\left(\pi \circ \widetilde{F}_{t}^{\prime}\right)=\pi_{*} \frac{\partial}{\partial t} \widetilde{F}_{t}^{\prime}=\pi_{*} \widetilde{H}^{\prime} .
$$

Corollary 3.2 says that $\widetilde{F}_{t}^{\prime}$ is $G$-invariant, like its initial data $F_{0}^{\prime}$, therefore $\pi_{*} \widetilde{H}^{\prime}=\widetilde{H}$. Then $\widetilde{F}_{t}$ is the evolution of initial data $F_{0}$, that is, $\widetilde{F}_{t}(\mathcal{B})=F_{t}(\mathcal{B})$ for any time $t$.

Remark 3.3 If the fibers are not closed we do not know if the solution of the mean curvature flow of the lift is unique, but if they are minimal, the same proof given for Theorem 1.1 shows that the lift of the mean curvature flow is, in any case, a $G$-invariant solution of the mean curvature flow. In the same way the projection of a $G$-invariant solution is again an evolution by mean curvature. Therefore if the projection of the initial data $\mathcal{M}_{0}$ is a closed submanifold $\mathcal{B}_{0}$ then there exists only one $G$-invariant solution of initial data $\mathcal{M}_{0}$.

\section{Examples and applications}

One of the best known examples of submersions is the family of the Hopf fibrations. Let $\mathbb{K}$ be one either the field $\mathbb{C}$ or the associative algebra $\mathbb{H}$ and $a$ be the real dimension of $\mathbb{K}$. We denote with $\mathbb{S}^{n}(c)$ the $n$-dimensional sphere with the canonical metric of constant curvature $c>0$. The action $T: \mathbb{S}^{a-1}(1) \times \mathbb{S}^{n a+a-1}(c) \rightarrow \mathbb{S}^{n a+a-1}(c),(\lambda, z) \mapsto \lambda z$ is by isometries which act transitively on the fiber. The Hopf fibrations are $\pi: \mathbb{S}^{n a+a-1}(c) \rightarrow \mathbb{K} \mathbb{P}^{n} \equiv \mathbb{S}^{n a+a-1} / \mathbb{S}^{a-1}$, 
$z \mapsto[z]$, where $[z]$ is the class of $z$ under the action $T$. The Riemannian metric that we consider on $\mathbb{K} \mathbb{P}^{n}$ is the one induced by the metric of $\mathbb{S}^{n a+a-1}(c)$ such that $\pi$ becomes a Riemannian submersion. For $\mathbb{K}=\mathbb{C}$ it is the well-known Fubini-Study metric.

Let us consider first the Hopf fibration $\pi: \mathbb{S}^{2 n+1} \rightarrow \mathbb{C P}^{n}$. In this case $V=J v$ is the vertical unit vector field, where $J$ is the complex structure of $\mathbb{C}^{n+1}$ and $v$ is the outward normal unit vector field of the sphere as a submanifold of $\mathbb{R}^{2 n+2} \equiv \mathbb{C}^{n+1}$. Let $\mathcal{B}_{0}$ be a submanifold of $\mathbb{C P}$ of dimension $m$ and codimension $k$ and $\mathcal{M}_{0}$ its lift to $\mathbb{S}^{2 n+1}$. The fibers $\mathbb{S}^{1}$ are geodesics, hence of course minimal. For every $i, J\left(\xi_{i}\right)^{\mathscr{H}}$ is tangent to the sphere and horizontal. Define $J\left(\xi_{i}\right)^{\mathscr{H}}=-U_{i}+N_{i}$ where $U_{i}$ is the component tangent to $\mathcal{M}_{0}$, while $N_{i}$ is normal. We want to compute the mixed terms in (2.4) for this submersion. As shown in [13], for every horizontal lift we have

$$
\mathcal{A}_{X} \mathscr{H} V=J(X)^{\mathscr{H}} .
$$

If $X$ is tangent to $\mathcal{B}_{0}$ then $A^{\prime}\left(X^{\mathscr{H}}, V\right)$ is a horizontal vector field and, by Lemma 2.2 and some trivial computation

$$
A^{\prime}\left(X^{\mathscr{H}}, V\right)=\sum_{i} \bar{g}\left(X^{\mathscr{H}}, U_{i}\right) \xi_{i}^{\mathscr{H}}
$$

Moreover since the fibers are geodesic curves, $A^{\prime}(V, V)=0$; together with (2.2), which holds in general, we have

$$
\left|A^{\prime}\right|^{2}=|A|^{2}+2 \sum_{i}\left|U_{i}\right|^{2}
$$

The canonical variation of the Hopf fibration gives a family $\left\{\bar{g}_{\lambda}\right\}_{\lambda>0}$ of metrics on $\mathbb{S}^{2 n+1}$. With respect to this metric, a unit vertical vector field is $V_{\lambda}=\lambda^{-\frac{1}{2}} J v$. With the same computation seen above we have that

$$
\left|A^{\prime}\right|^{2}=|A|^{2}+2 \lambda^{-\frac{1}{2}} \sum_{i}\left|U_{i}\right|^{2} .
$$

Then, for any $\lambda>0$

$$
|A|^{2} \leq\left|A^{\prime}\right|^{2} \leq|A|^{2}+2 \lambda^{-\frac{1}{2}} \operatorname{cod} \mathcal{M}_{0}=|A|^{2}+2 \lambda^{-\frac{1}{2}} \operatorname{cod}_{0}
$$

holds. Obviously since $H^{\prime}$ and $H$ are $\pi$-related we have that $\left|H^{\prime}\right|^{2}=|H|^{2}$ in any case.

In the same way we can study the Hopf fibration $\pi: \mathbb{S}^{4 n+3} \rightarrow \mathbb{H} \mathbb{P}^{n}$. The fibers are $\mathbb{S}^{3}$ which are totally geodesic. Let $J_{1}, J_{2}$ and $J_{3}$ be the complex structures of $\mathbb{H}^{n+1}$ given by the multiplication of the quaternionic imaginary units. Then $\left(V_{1}=J_{1} v, V_{2}=J_{2} v, V_{3}=J_{3} v\right)$ is an orthonormal basis of $\mathscr{V}$. Following the same notations and the same computations of the previous case, we define for every $i$ and $\alpha J_{\alpha} \xi_{i}^{\mathscr{H}}=-U_{i \alpha}+N_{i \alpha}$ where $U_{i \alpha}$ is tangent to $\mathcal{M}_{0}$, while $N_{i \alpha}$ is normal. Moreover

$$
\mathcal{A}_{X} \mathscr{H} V_{\alpha}=J_{\alpha}(X)^{\mathscr{H}},
$$

and for every $\alpha$ and $\beta$

$$
\begin{aligned}
A^{\prime}\left(X^{\mathscr{H}}, V_{\alpha}\right) & =\sum_{i} \bar{g}\left(X^{\mathscr{H}}, U_{i \alpha}^{\mathscr{H}}\right) \xi_{i}^{\mathscr{H}}, \\
A^{\prime}\left(V_{\alpha}, V_{\beta}\right) & =0 .
\end{aligned}
$$


Then we get

$$
\left|A^{\prime}\right|^{2}=|A|^{2}+2 \sum_{i, \alpha}\left|U_{i \alpha}^{\mathscr{H}}\right|^{2}=|A|^{2}+2 \sum_{i, \alpha}\left|U_{i \alpha}\right|^{2} .
$$

The canonical variation of this Hopf fibration gives a second family $\left\{\tilde{g}_{\lambda}\right\}_{\lambda>0}$ of metric on $\mathbb{S}^{4 n+3}$. Similarly to the previous case we have

$$
\left|A^{\prime}\right|^{2}=|A|^{2}+2 \lambda^{-\frac{1}{2}} \sum_{i, \alpha}\left|U_{i \alpha}\right|^{2} .
$$

Then for every $\lambda>0$

$$
|A|^{2} \leq\left|A^{\prime}\right|^{2} \leq|A|^{2}+6 \lambda^{-\frac{1}{2}} \operatorname{cod} \mathcal{M}_{0}=|A|^{2}+6 \lambda^{-\frac{1}{2}} \operatorname{cod} \mathcal{B}_{0} .
$$

From the application of Theorem 1.1 we obtain the following results:

Proposition 4.1 Let $\mathcal{M}_{0}$ be a closed $\mathbb{S}^{1}$-invariant hypersurface of $\left(\mathbb{S}^{2 n+1}, \bar{g}_{\lambda}\right)$, with $n \geq 3$. If $\mathcal{M}_{0}$ satisfies

$$
\left|A^{\prime}\right|^{2}<\frac{1}{2 n-2}\left|H^{\prime}\right|^{2}+2+2 \lambda^{-\frac{1}{2}}
$$

then the mean curvature flow of $\mathcal{M}_{0}$ develops a singularity in finite time and converges to an $\mathbb{S}^{1}$, hence such an $\mathcal{M}_{0}$ is diffeomorphic to a $\mathbb{S}^{1} \times \mathbb{S}^{2 n-1}$.

Proof Since $\mathcal{M}_{0}$ is $\mathbb{S}^{1}$ invariant we can project it with the Hopf fibration to a hypersurface $\mathcal{B}_{0}$ of $\mathbb{C P}^{n}$. For hypersurfaces we have necessarily $\left|A^{\prime}\right|^{2}=|A|^{2}+2 \lambda^{-\frac{1}{2}}$. Then $\mathcal{B}_{0}$ satisfies

$$
|A|^{2}<\frac{1}{2 n-2}|H|^{2}+2
$$

By Theorem 1.1 of [15] the evolution of $\mathcal{B}_{0}$ converges in finite time to a round point $p$. By Theorem 1.1 we have that the evolution of $\mathcal{M}_{0}$ converges in finite time to the lift of this point, which is a fiber.

Note that Proposition 1.2 is a particular case of Proposition 4.1 obtained with $\lambda=1$.

For higher codimension we have the following result.

Proposition 4.2 Consider $\mathcal{M}_{0}$ a closed $\mathbb{S}^{1}$-invariant submanifold of $\left(\mathbb{S}^{2 n+1}, \bar{g}_{\lambda}\right)$ of dimension $m$ and codimension $2 \leq k<\frac{2 n-3}{5}$ satisfying the pinching condition

$$
|A|^{2}<\frac{1}{m-2}|H|^{2}+\frac{m-4-4 k}{m-1} .
$$

Then either

1) the evolution of $\mathcal{M}_{0}$ converges in finite time to an $\mathbb{S}^{1}$,

or

2) the evolution of $\mathcal{M}_{0}$ is defined for any time $0 \leq t<\infty$ and converges to a smooth totally geodesic submanifold, which is an $\mathbb{S}^{2 n-k+1}$.

If $k$ is odd only case 1) can occur. 
Proof The proof is the same as that of the previous Proposition: it follows again from Theorem 1.1 of [15] and Theorem 1.1: using the inequality $|A|^{2} \leq\left|A^{\prime}\right|^{2}$ we have that $\mathcal{B}_{0}=\pi\left(\mathcal{M}_{0}\right)$ satisfies the same pinching inequality (4.2) and the thesis follows since

$$
\pi^{-1}\left(\mathbb{C P}^{n-\frac{k}{2}}\right)=\mathbb{S}^{2 n-k+1} .
$$

This time let us consider the canonical deformation of the Hopf fibration $\pi: \mathbb{S}^{4 n+3} \rightarrow \mathbb{H} \mathbb{P}^{n}$ (for $\lambda=1$ we have Proposition 1.3).

Proposition 4.3 Let $\mathcal{M}_{0}$ be a closed $\mathbb{S}^{3}$-invariant hypersurface of $\left(\mathbb{S}^{4 n+3}, \tilde{g}_{\lambda}\right)$, with $n \geq 3$. If $\mathcal{M}_{0}$ satisfies

$$
\left|A^{\prime}\right|^{2}<\frac{1}{4 n-2}\left|H^{\prime}\right|^{2}+2+6 \lambda^{-\frac{1}{2}}
$$

then the mean curvature flow of $\mathcal{M}_{0}$ develops a singularity in finite time and converges to an $\mathbb{S}^{3}$. It follows that such an $\mathcal{M}_{0}$ is diffeomorphic to a $\mathbb{S}^{3} \times \mathbb{S}^{4 n-1}$.

Proof For hypersurfaces we have $\left|A^{\prime}\right|^{2}=|A|^{2}+6 \lambda^{-\frac{1}{2}}$, hence $\mathcal{B}_{0}=\pi\left(\mathcal{M}_{0}\right)$ satisfies $|A|^{2}<\frac{1}{4 n-2}|H|^{2}+2$, then by Theorem 7.1 of [15] the evolution of $\mathcal{B}_{0}$ shrinks to a round point in finite time. The thesis follows as in the previous propositions.

A further example is given by the submersion $\rho: \mathbb{C P}^{2 n+1} \rightarrow \mathbb{H} \mathbb{P}^{n}$ described in [5]: it is the submersion that makes the following diagrams commutative

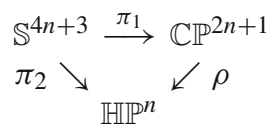

where $\pi_{1}$ and $\pi_{2}$ are the usual Hopf fibrations. The fibers of $\rho$ are $\mathbb{C P}^{1} \equiv \mathbb{S}^{2}(4)$ and hence they are totally geodesic. Lifting a hypersurface of $\mathbb{H} \mathbb{P}^{n}$ to a hypersurface of $\mathbb{C P}^{2 n+1}$ via $\rho$ we have that $\left|A^{\prime}\right|^{2}=|A|^{2}+4$. In the same way as the previous propositions we can prove the following result.

Proposition 4.4 Let $\mathcal{M}_{0}$ be a closed $\mathbb{C P}^{1}$-invariant hypersurface of $\mathbb{C P}^{2 n+1}$. If $\mathcal{M}_{0}$ satisfies

$$
|A|^{2}<\frac{1}{4 n-2}|H|^{2}+6
$$

then the mean curvature flow of $\mathcal{M}_{0}$ develops a singularity in finite time and converges to a fiber $\mathbb{C P}^{1}$, and therefore such an $\mathcal{M}_{0}$ is diffeomorphic to a $\mathbb{S}^{2} \times \mathbb{S}^{4 n-1}$.

The examples seen before are all principal bundles with compact fibers. An interesting case with non-compact fibers comes from the Heisenberg group $\mathbb{H}^{n}$ (not to be confused with the algebra of quaternions!). The Heisenberg group is the Lie group $\mathbb{R}^{2 n} \times \mathbb{R}$ endowed with the following product:

$$
(x, y, z)\left(x^{\prime}, y^{\prime}, z^{\prime}\right)=\left(x+x^{\prime}, y+y^{\prime}, z+z^{\prime}+\frac{1}{2}\left(\left\langle x, y^{\prime}\right\rangle-\left\langle y, x^{\prime}\right\rangle\right)\right),
$$


where $x, x^{\prime}, y, y^{\prime} \in \mathbb{R}^{n}, z, z^{\prime} \in \mathbb{R}$ and $\langle\cdot, \cdot\rangle$ is the Euclidean scalar product of $\mathbb{R}^{n}$. With respect to the coordinates $(x, y, z)=\left(x_{1}, \ldots, x_{n}, y_{1}, \ldots, y_{n}, z\right)$ we define the following left invariant vector fields on $\mathbb{H}^{n}$ :

$$
X_{j}=\frac{\partial}{\partial x_{j}}-\frac{1}{2} y_{j} \frac{\partial}{\partial z}, \quad Y_{j}=\frac{\partial}{\partial y_{j}}+\frac{1}{2} x_{j} \frac{\partial}{\partial z}, \quad V=\frac{\partial}{\partial z} .
$$

Declaring as orthonormal the basis $\left(X_{j}, Y_{j}, V\right)_{j}$ we have a left-invariant metric $\bar{g}$ on $\mathbb{H}^{n}$. On $\mathbb{C}^{n}$ consider the Euclidean metric: then

$$
\pi:(x, y, z) \in \mathbb{H}^{n} \mapsto(x+i y) \in \mathbb{C}^{n}
$$

is a Riemannian submersion. The fibers are the vertical lines:

$$
\pi^{-1}\left(x_{0}+i y_{0}\right)=\left\{\left(x_{0}, y_{0}, t\right) \mid t \in \mathbb{R}\right\} .
$$

Moreover $\mathscr{V}=\operatorname{span}\langle V\rangle$ and $\mathscr{H}=\operatorname{span}\left\langle X_{j}, Y_{j}\right\rangle_{j=1, \ldots, n}$. The structural group is the group of vertical translations, that is the multiplication by a point of the type $(0,0, t)$. It is a group of isometries and it is isomorphic to $(\mathbb{R},+)$. The Levi-Civita connection associated to $\bar{g}^{\prime}$ is determined by

$$
\begin{gathered}
\bar{\nabla}_{X_{j}} Y_{j}=-\bar{\nabla}_{Y_{j}} X_{j}=\frac{1}{2} V, \\
\bar{\nabla}_{X_{j}} V=\bar{\nabla}_{V} X_{j}=-\frac{1}{2} Y_{j} \\
\bar{\nabla}_{Y_{j}} V=\bar{\nabla}_{V} Y_{j}=\frac{1}{2} X_{j}
\end{gathered}
$$

and is zero for all others pairs of vector of the basis $\left(X_{j}, Y_{j}, V\right)_{j=1, \ldots, n}$. A proof can be found in [11]. In particular $\bar{\nabla}_{V} V$ vanishes, hence the fibers of $\pi$ are geodesics. On the horizontal distribution $\mathscr{H}$ we have a complex structure $J$ defined on the vector of the basis by $J X_{j}=Y_{j}$ and $J Y_{j}=-X_{j}$ for all $j$. Then, more succinctly, for any horizontal vector field $Z$ on $\mathbb{H}^{n}$ we have

$$
\bar{\nabla}_{Z} V=\bar{\nabla}_{V} Z=-J Z .
$$

Now consider $\mathcal{B}_{0}$ a submanifold of the Euclidean space $\mathbb{C}^{n}$ of dimension $m$ and codimension $k$. Its lift via $\pi$ is a submanifold $\mathcal{M}_{0}$ invariant with respect to vertical translations. Using notation 2.3, by (4.4) and some trivial computations we have:

$$
A^{\prime}\left(X_{j}^{\mathscr{H}}, V\right)=\frac{1}{2} \sum_{\alpha=1}^{k} \bar{g}\left(X_{j}^{\mathscr{H}}, J \xi_{\alpha}^{\mathscr{H}}\right) \xi_{\alpha}^{\mathscr{H}}=\frac{1}{2} \sum_{\alpha=1}^{k} \bar{g}\left(X_{j}, J \xi_{\alpha}\right) \xi_{\alpha}^{\mathscr{H}},
$$

where the $J$ in the last term is the usual complex structure of $\mathbb{C}^{n}$. This result is very similar to what we have for Hopf fibration. It follows that

$$
\left|A^{\prime}\right|^{2}=|A|^{2}+\frac{1}{2} \sum_{\alpha=1}^{k}\left|J \xi_{\alpha}^{\top}\right|^{2}
$$

therefore

$$
|A|^{2} \leq\left|A^{\prime}\right|^{2} \leq|A|^{2}+\frac{k}{2},
$$

with $\left|A^{\prime}\right|^{2}=|A|^{2}$ if and only if for every $\alpha J \xi_{\alpha}$ is normal to $\mathcal{B}_{0}$ (in this case $\mathcal{B}_{0}$ is a complex submanifold of $\mathbb{C}^{n}$ ), while $\left|A^{\prime}\right|^{2}=|A|^{2}+\frac{k}{2}$ if and only if for every $\alpha J \xi_{n+\alpha}$ is tangent 
to $\mathcal{B}_{0}$ (that is, $\mathcal{B}_{0}$ is CR-submanifold of $\mathbb{C}^{n}$ of CR-dimension $m-k$ ). In the first case, in particular, $\mathcal{B}_{0}$ is a minimal submanifold. The classical Huisken's result [8] about the evolution of convex hypersurfaces of the Euclidean space gives the following result for hypersurfaces of the Heisenberg group.

Proposition 4.5 Let $\mathcal{M}_{0}$ be a hypersurface of $\mathbb{H}^{n}$. If $\mathcal{M}_{0}$ is a cylinder with vertical axis, without boundary and its projection via $\pi$ is a convex hypersurface of $\mathbb{R}^{2 n}$, then there is a unique solution of the mean curvature flow of $\mathcal{M}_{0}$ invariant with respect to vertical translations. Moreover this solution develops a singularity in finite time and converges to a vertical line. Therefore such an $\mathcal{M}_{0}$ is diffeomorphic to a cylinder $\mathbb{S}^{2 n-1} \times \mathbb{R}$.

Proof Such an $\mathcal{M}_{0}$ is invariant with respect to vertical translations. The fiber of $\pi$ are not closed so we can apply Theorem 1.1 as we did in Remark 3.3. Let $\mathcal{B}_{0}=\pi\left(\mathcal{M}_{0}\right)$. By the main result of [8], $\mathcal{B}_{0}$ shrinks to a round point in finite time. The thesis follows lifting this result to $\mathcal{M}_{0}$.

Using the main theorem of [1], we have the following result for submanifolds of arbitrary codimension in the Heisenberg group.

Proposition 4.6 Let $\mathcal{M}_{0}$ be a cylinder with vertical axis of $\mathbb{H}^{n}$ of dimension $m \geq 3$, without boundary and whose horizontal section is a closed submanifold. If $\mathcal{M}_{0}$ has $H \neq 0$ everywhere and satisfies $|A|^{2} \leq c|H|^{2}$ with

$$
c \leq\left\{\begin{array}{lll}
\frac{4}{3(m-1)} & \text { if } & 3 \leq m \leq 5, \\
\frac{1}{m-2} & \text { if } & m>5,
\end{array}\right.
$$

then the mean curvature flow of initial data $\mathcal{M}_{0}$ has a unique $\mathbb{R}$-invariant solution and this solution converges in finite time to a vertical line. Hence such an $\mathcal{M}_{0}$ is diffeomorphic to a cylinder $\mathbb{S}^{m-1} \times \mathbb{R}$.

Proof We have that $\mathcal{B}_{0}=\pi\left(\mathcal{M}_{0}\right)$ is a closed submanifold of $\mathbb{R}^{2 n}$ of dimension $m-1$. By (4.5), $\mathcal{B}_{0}$ satisfies

$$
|A|^{2} \leq\left|A^{\prime}\right|^{2} \leq c\left|H^{\prime}\right|^{2}=c|H|^{2} .
$$

The main result of [1] says that the evolution by mean curvature of $\mathcal{B}_{0}$ shrinks to a point in finite time. We can apply Theorem 1.1 to the unique $\mathbb{R}$-invariant solution obtaining the convergence of $\mathcal{M}_{0}$ to a fiber of $\pi$, which is a vertical line of $\mathbb{H}^{n}$.

Another interesting submersion is the one that arises with the tangent sphere bundle of a Riemannian manifold equipped with the Sasaki metric. For any Riemannian manifold $(\overline{\mathcal{B}}, \bar{g})$, let $T \overline{\mathcal{B}}$ be its tangent bundle and for any $r>0$ let $T^{r} \overline{\mathcal{B}}=\left\{\left.(p, u) \in T \overline{\mathcal{B}}|| u\right|_{\bar{g}}=r\right\}$ be the tangent sphere bundle of radius $r$. The natural projection

$$
\pi:(p, u) \in T^{r} \overline{\mathcal{B}} \mapsto p \in \overline{\mathcal{B}}
$$

is a submersion. In this special case, for any vector field $X$ on $\overline{\mathcal{B}}$ we can also define a lift $X^{\mathscr{T}} \in \mathscr{V}$ called tangent lift: see [10] for an exhaustive description. The Sasaki metric is a natural metric $\bar{g}$ on $T \overline{\mathcal{B}}$, which, when restricted to $T^{r} \overline{\mathcal{B}}$, has the following form:

$$
\begin{aligned}
\bar{g}_{(p, u)}\left(X^{\mathscr{H}}, Y^{\mathscr{H}}\right) & =\bar{g}_{p}(X, Y), \\
\bar{g}_{(p, u)}\left(X^{\mathscr{T}}, Y^{\mathscr{T}}\right) & =\bar{g}_{p}(X, Y)-\frac{1}{r^{2}} \bar{g}_{p}(X, u) \bar{g}_{p}(Y, u), \\
\bar{g}_{(p, u)}\left(X^{\mathscr{H}}, Y^{\mathscr{T}}\right) & =0,
\end{aligned}
$$


for any $X$ and $Y$ tangent to $\overline{\mathcal{B}}$. With this metric the projection $\pi: T^{r} \overline{\mathcal{B}} \rightarrow \overline{\mathcal{B}}$ is a Riemannian submersion with fibers $\pi^{-1}(p)=T_{p}^{r} \overline{\mathcal{B}}$, i.e. the sphere of radius $r$ tangent to $\overline{\mathcal{B}}$ in $p$. The horizontal distribution of $\pi$ is generated by the horizontal lifts and the vertical distribution is generated by the tangential lifts. The group of isometries that we are considering acts only on the vectorial part as an isometry of $T_{p}^{r} \overline{\mathcal{B}}$ and is isomorphic to $O(n)$, where $n$ is the dimension of $\overline{\mathcal{B}}$. Note that in this case the action of the group is not free - in fact the orbits are not isometric to the group - but the quotient manifold $T^{r} \overline{\mathcal{B}} / O(n) \equiv \overline{\mathcal{B}}$ is a well defined manifold. The Levi-Civita connection of the Sasaki metric on $T^{r} \overline{\mathcal{B}}$ is

Lemma 4.7 [10] Let $X$ and $Y$ be vector fields tangent to $\overline{\mathcal{B}}$, then we have:

1) $\left(\bar{\nabla}_{X} \mathscr{H} Y^{\mathscr{H}}\right)_{(p, u)}=\left(\bar{\nabla}_{X} Y\right)_{(p, u)}^{\mathscr{H}}-\frac{1}{2}\left(\bar{R}_{p}(X, Y) u\right)^{\mathscr{T}}$,

2) $\left(\bar{\nabla}_{X} \mathscr{H} Y^{\mathscr{T}}\right)_{(p, u)}=\left(\bar{\nabla}_{X} Y\right)^{\mathscr{T}}{ }_{(p, u)}+\frac{1}{2}\left(\bar{R}_{p}(u, Y) X\right)^{\mathscr{H}}$,

3) $\left(\bar{\nabla}_{X} \mathscr{T} Y^{\mathscr{H}}\right)_{(p, u)}=\frac{1}{2}\left(\bar{R}_{p}(u, X) Y\right)^{\mathscr{H}}$,

4) $\left(\bar{\nabla}_{X} \mathscr{T} Y^{\mathscr{T}}\right)_{(p, u)}=-\frac{1}{r^{2}} \bar{g}_{p}(u, Y) X^{\mathscr{T}}$,

where $\bar{R}$ is the Riemann curvature tensor of $\overline{\mathcal{B}}$.

The fibers are closed and the last equation shows that they are also totally geodesic: $\hat{A}\left(X^{\mathscr{T}}, Y^{\mathscr{T}}\right)$ is the horizontal part of $\bar{\nabla}_{X} \mathscr{T} Y^{\mathscr{T}}$. From now on let us consider a submanifold $\mathcal{B}_{0}$ of dimension $n$ and codimension $k$ and $\mathcal{M}_{0}$ its $O(n+k)$-invariant lift to $T^{r} \overline{\mathcal{B}}$. Since, in this case, we have a way to lift vector fields on $\overline{\mathcal{B}}$ to vector fields tangent to the fibers, we modify notation 2.3. For any $p \in \mathcal{B}_{0}$ and any $(p, u) \in \pi^{-1}\{p\}$, let $\left(X_{1}, \ldots, X_{n}\right)$ be an orthonormal basis tangent to $\mathcal{B}_{0}$ in $p$ and $\left(\xi_{1}, \ldots, \xi_{k}\right)$ an orthonormal basis normal to $\mathcal{B}_{0}$ in $p$ such that

$$
u=r \cos (\vartheta) X_{1}+r \sin (\vartheta) \xi_{1},
$$

for some $\vartheta$. Let $Z=\sin (\vartheta) X_{1}-\cos (\vartheta) \xi_{1}$ : then $\left(u, Z, X_{2}, \ldots, X_{n}, \xi_{2}, \ldots, \xi_{k}\right)$ is an orthogonal basis of $T_{p} \mathcal{B}$. By (4.6) we have that

$$
\left(X_{1}^{\mathscr{H}}, \ldots, X_{n}^{\mathscr{H}}, Z^{\mathscr{T}}, X_{2}{ }^{\mathscr{T}}, \ldots, X_{n}{ }^{\mathscr{T}}, \xi_{2}{ }^{\mathscr{T}}, \ldots, \xi_{k}{ }^{\mathscr{T}}\right)
$$

is an orthonormal basis tangent to $\mathcal{M}_{0}$ in $(p, u)$, while $\left(\xi_{1}^{\mathscr{H}}, \ldots, \xi_{k}^{\mathscr{H}}\right)$ is an orthonormal basis normal to $\mathcal{M}_{0}$ in $(p, u)$. As a concrete example, consider $\overline{\mathcal{B}}=\mathbb{S}^{n+k}(c)$, the sphere of constant curvature $c>0$. By Lemma 4.7 we have

$$
A^{\prime}\left(X_{i}^{\mathscr{H}}, Z^{\mathscr{T}}\right)(p, u)=\frac{1}{2} \sum_{\alpha=1}^{k} \bar{R}_{p}\left(u, Z, X_{i}, \xi_{\alpha}\right) \xi_{\alpha}^{\mathscr{H}}=-\frac{c r}{2} \delta_{i 1} \xi_{1}^{\mathscr{H}} .
$$

Similarly

$$
\begin{aligned}
A^{\prime}\left(X_{i}^{\mathscr{H}}, X_{j}{ }^{\mathscr{T}}\right)(p, u) & =-\frac{c r}{2} \sin (\vartheta) \delta_{i j} \xi_{1}^{\mathscr{H}} \\
A^{\prime}\left(X_{i}^{\mathscr{H}}, \xi_{j}{ }^{\mathscr{T}}\right)(p, u) & =\frac{c r}{2} \cos (\vartheta) \delta_{i 1} \xi_{j}^{\mathscr{H}} .
\end{aligned}
$$

Then

$$
\begin{aligned}
\left|A^{\prime}\right|^{2}(p, u) & =|A|^{2}(p)+\frac{c^{2} r^{2}}{2}\left(1+(n-1) \sin ^{2}(\vartheta)+(k-1) \cos ^{2}(\vartheta)\right) \\
& =|A|^{2}(p)+\frac{c^{2}}{2}\left(r^{2}+(n-1)\left|u^{\perp}\right|^{2}+(k-1)\left|u^{\top}\right|^{2}\right)
\end{aligned}
$$


where $\perp$ (respectively $T$ ) indicates the normal (respectively the tangent) component with respect to $\mathcal{B}_{0}$. In particular we have

$$
|A|^{2}(p)+\frac{c^{2} r^{2}}{2} \min \{k, n\} \leq\left|A^{\prime}\right|^{2}(p, u) \leq|A|^{2}(p)+\frac{c^{2} r^{2}}{2} \max \{k, n\} .
$$

Lifting the submanifolds of the sphere considered by Huisken [9] and Baker [2] we have the following result as a consequence of Theorem 1.1.

Proposition 4.8 For any $r>0, n \geq 3$ and $k \geq 1$, let $\mathcal{M}_{0}$ be an $O(n+k)$-invariant submanifold of $T^{r} \mathbb{S}^{n+k}(c)$ of dimension $2 n+k-1$. Suppose that $\mathcal{M}_{0}$ satisfies the pinching condition

$$
|A|^{2}<\frac{1}{n-1}|H|^{2}+2 c+\frac{c^{2}}{2} \min \{k, n\},
$$

then the mean curvature flow with initial data $\mathcal{M}_{0}$ converges in finite time to a fiber $\pi^{-1}(p)=$ $T_{p}^{r} \mathbb{S}^{n+k}(c)$ or the flow is defined for any time and converges to $\pi^{-1}\left(\mathbb{S}^{n}(c)\right)$, which is a minimal, but not totally geodesic, submanifold of $T^{r} \mathbb{S}^{n+k}(c)$.

Open Access This article is distributed under the terms of the Creative Commons Attribution 4.0 International License (http://creativecommons.org/licenses/by/4.0/), which permits unrestricted use, distribution, and reproduction in any medium, provided you give appropriate credit to the original author(s) and the source, provide a link to the Creative Commons license, and indicate if changes were made.

\section{References}

1. Andrews, B., Baker, C.: Mean curvature flow of pinched submanifolds to spheres. J. Differ. Geom. 85, 357-395 (2010)

2. Baker, C.: The mean curvature flow of submanifolds of high codimension. Ph.D. thesis. Australian National University. arXiv:1104.4409v1 [math.DG] (2011)

3. Besse, A.L.: Einstein Manifolds. Springer, Hidelberg (1987)

4. Escobales Jr., R.H.: Riemannian submersions with totally geodesic fibers. J. Differ. Geom. 10, 253-276 (1975)

5. Escobales Jr., R.H.: Riemannian submersions from complex projective space. J. Differ. Geom. 13, 93-107 (1978)

6. Falcitelli, M., Ianus, S., Pastore, A.M.: Riemannian Submersions and Related Topics. World Scientific, River Edge, NJ (2004)

7. Gudmundsson, S., Kappos, E.: On the geometry of tangent bundles. Expos. Math. 20, 1-41 (2002)

8. Huisken, G.: Flow by mean curvature of convex surfaces into spheres. J. Differ. Geom. 20, 237-266 (1984)

9. Huisken, G.: Deforming hypersurfaces of the sphere by their mean curvature. Math. Z. 195, 205-219 (1987)

10. Kowalski, O., Sekizawa, M.: On tangent sphere bundles with small or large constant radius. Ann. Glob. Anal. Geom. 18, 207-219 (2000)

11. Marenich, V.: Geodesics in Heisenberg groups. Geom. Dedicata 66(2), 175-185 (1997)

12. Nguyen, H.T.: Convexity and cylindrical estimates for mean curvature flow in the sphere. Trans. Am. Math. Soc. 367, 4517-4536 (2015)

13. O’Neill, B.: The fundamental equations of a submersion. Mich. Math. J. 13, 459-469 (1966)

14. Pacini, T.: Mean curvature flow, orbits, moment maps Trans. Am. Math. Soc. 355(8), 3343-3357 (2003)

15. Pipoli, G., Sinestrari, C.: Mean curvature flow of pinched submanifolds of $\mathbb{C P}^{n}$, arXiv:1502.00519 [math.DG]

16. Smoczyk, K.: Symmetric hypersurfaces in Riemannian manifolds contracting to Lie-groups by their mean curvature. Calc Var Partial Differ Equ 4(2), 155-170 (1996) 